\title{
Exchange parameters in Fe-based molecular magnets
}

\author{
A. V. Postnikov* \\ Universität Osnabrück - Fachbereich Physik, D-49069 Osnabrück ${ }^{1}$ \\ G. Bihlmayer and S. Blügel \\ Institut für Festkörperforschung, Forschungszentrum Jülich, D-52425 Jülich, \\ Germany
}

\begin{abstract}
The calculation of interatomic magnetic exchange interactions entering the Heisenberg model from the standpoint of the density functional theory (DFT) is outlined for two Fe-based molecular magnets: a trinuclear complex with a Schiff base ligand, which makes an antiferromagnetically coupled frustrated system, and a model bipyrimidine-connected planar network of Fe ions. First-principles electronic structure calculations are performed using the real-space method SIESTA and the fullpotential linearized augmented plane wave FLAPW method FLEUR, correspondingly. We discuss the application of fixed spin moment technique for preparing the system in a given magnetic configuration, and the effect of intraatomic Coulomb correlation, approximated by the $\mathrm{LDA}+U$ technique, on the values of interaction parameters.
\end{abstract}

Key words: Ab initio calculations, single-molecule magnets, magnetic interactions PACS: 31.15.Ew, 31.70.Ks, 71.15.Nc, 71.20.Rv, 71.70.Gm, 75.50.Xx

\section{Introduction}

Since the first systematizations of their chemistry and basic properties by, e.g., Kahn in 1993 [1], molecular magnets are now developing into a promising class of magnetic nanomaterials with a great potential in applications in areas such

* Corresponding author. Tel.: +49-541-9692377; fax: -9692351.

Email address: apostnik@uos.de (A. V. Postnikov).

1 Permanent address: Institute of Metal Physics, 620219 Yekaterinburg, Russia 
as magnetic storage, quantum computing, or magneto-optical devices. An upto-date review on microscopic properties (spin density, exchange interactions, magnetic anisotropy) of molecular magnets accessible in experiment and from first-principles calculations, is going to appear in Ref. [2]. Its major part is summarized as an internet publication [3]. In the present work we address the issue of extracting the interatomic exchange interaction parameters entering the Heisenberg model form the density functional theory (DFT). We discuss two chemical systems of different complexity. The actual calculations are done by two different methods, SIESTA [4] using compact and strictly confined atom-centered basis functions (see Ref. [5] for details), and the FLEUR [6] code, a realization of the highly accurate full-potential linearized augmented plane wave (FLAPW) method. Both calculations used the generalized gradient approximation to the exchange-correlation functionals.

We skip the subtleties in the formulation of exchange parameters within the DFT; a detailed discussion can be found in Ref. [3]. Basically, we refer to total energies in different specially prepared magnetic configurations, calculated from first principles. Sec. 2 covers the extraction of meaningful results for a Fe-trinuclear system by applying the fixed spin moment formalism. Sec. 3 addresses a model Fe-binuclear system and, specifically, the importance of intraatomic correlation, treated beyond the conventional DFT formalism.

\section{Fixed Spin Moment treatment of a Fe-trinuclear system}

One of the ways to arrive at the values of interaction parameters in a magnetic system whose properties are well decribed by the Heisenberg model is to compare total energies for different magnetic configurations. This is rather straightforward if, say, ferromagnetic (FM) and antiferromagnetic (AFM) states are well defined and at least metastable. A more complicated case is a frustrated system, an example of which is made of three Fe atoms incorporated into organic molecule. We consider specifically $\mathrm{Fe}_{3}(\mathrm{OAc})_{3} \mathrm{~L}_{3}$, a synthesized by Boskovic et al. [7] derivate of the Schiff base $\mathrm{H}_{2} \mathrm{~L}=$ salicylidene-2-ethanolamine. The molecular unit used in the electronic structure calculation by the SIESTA method is shown in Fig. 2 (a) of Ref. [7]. The magnetization measurements reported in Ref. [7] indicate an (almost AFM) ground state with the total spin $S=1 / 2$, coming about from the interaction of three $s=5 / 2$ spins of Fe(III) ions. Experimentally, the interaction parameters $J$ of the Heisenberg model, introduced in Ref. [7] as $H=-2 \sum_{\text {Fe pairs }} J_{i j} \mathbf{s}_{i} \mathbf{s}_{j}$, were reported to be necessarily different for an acceptable fitting of data, even as the molecule nearly maintains a trifold symmetry axis: $J_{i j}=-15.1 \mathrm{~K},-13.6 \mathrm{~K}$, and $-12.4 \mathrm{~K}$.

We proposed earlier [8] to use the Fixed Spin Moment (FSM) method [9] for selecting a magnetic configuration of a molecular magnet (a six-center "ferric wheel"), improving simultaneously the stability of the electronic structure calcu- 


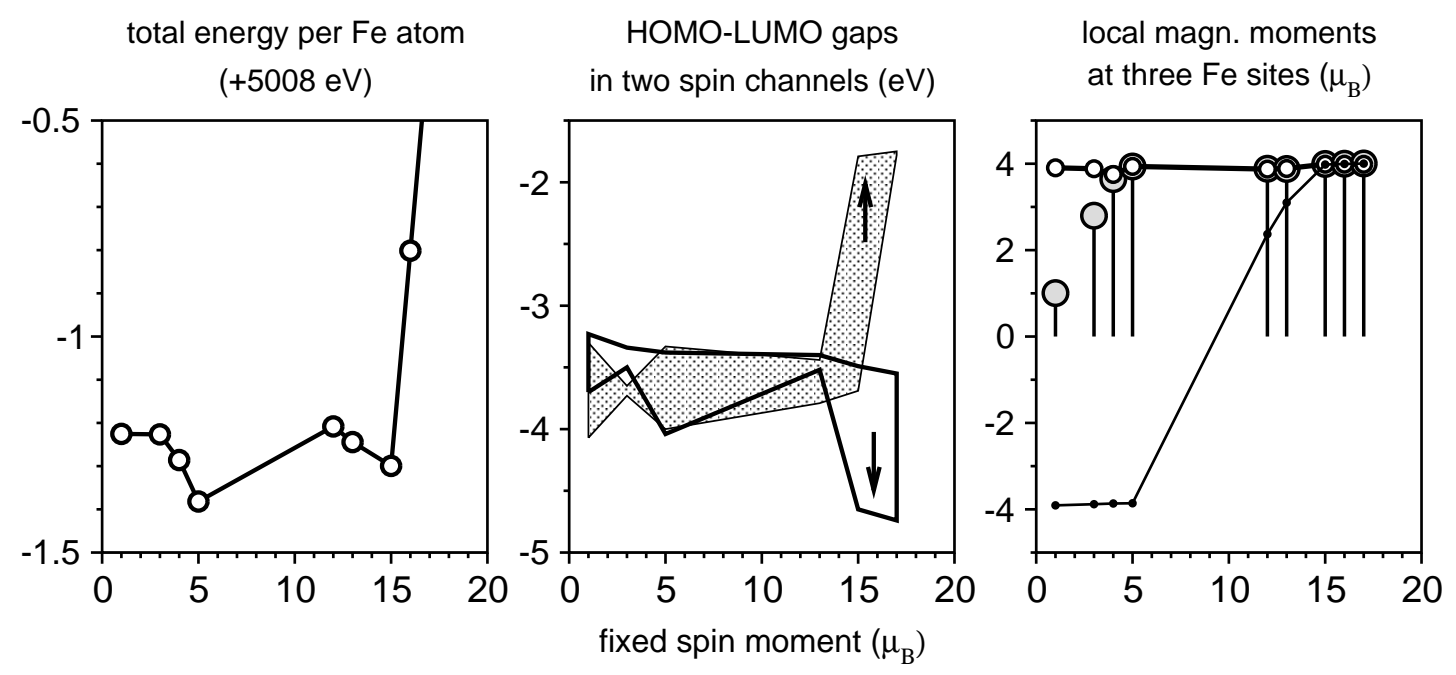

Fig. 1. Results of the FSM calculation for $\left[\mathrm{Fe}_{3}(\mathrm{OAc})_{3} \mathrm{~L}_{3}\right]$. See main text and explanations [19] for details.

lation. For the present system this idea becomes even more important, because it is no more obvious how to simulate a (nearly) AFM trial magnetic configuration in the DFT run, nor how to enforce an inversion of a selected local magnetic moment. On the contrary, by running a sequence of FSM calculations from the maximal nominal value of the total spin $S=15 / 2$ (and higher) down to zero, one can scan the variety of all possible magnetic solutions within the DFT.

The results of our FSM calculation are shown in Fig. 1. The total energy (left panel) has several local minima, the lowest one being for the FSM value of 5 $\mu_{\mathrm{B}}$. The right panel shows that this situation corresponds to two Fe magnetic moments aligned in parallel and the third one aligned in the opposite direction. A competitive energy minimum is found for FSM $=15 \mu_{\mathrm{B}}$, i.e., the ferromagnetic configuration. The solution with FSM $=1 \mu_{\mathrm{B}}$ has higher energy than the latter one by $74 \mathrm{meV}$, per Fe atom. It should be noted that, whereas the ground state of the system is experimentally found to have $S=1 / 2$, it must have a mixed nature over many quantum states, and hence does not correspond to any singledeterminant DFT result. Nevertheless, the tendency for antiparallel coupling of Fe magnetic moments is correctly provided by our calculation; moreover the order of magnitude of the interaction constants can be correctly recovered: consistently with the above formula for the Heisenberg Hamiltonian, $-J \approx\left(E^{\uparrow \uparrow}-E^{\uparrow \uparrow \downarrow}\right) /\left(8 s^{2}\right)$ amounts to $57 \mathrm{~K}$. The error (overestimation by a factor of about 4) in the DFT calculation is nearly the same as we found earlier for the "ferric wheel" [8]. A possible reason for such systematic discrepancy can be the underestimation of the intraatomic Coulomb correlation, to be discussed in the next Section.

Two issues might need further discussion. The HOMO-LUMO gaps shown in the middle panel of Fig. 1 are different in the FSM formalism for two spin directions, because the fixing of the total spin amounts to an imposition of an external magnetic field, moving apart the chemical potentials in two spin channels. Normally this would require the consideration of a Zeeman term when dealing with total 
energies. However, for a system with a common band gap in both spin channels a unique chemical potential can be found for both spin directions, therefore the FSM scheme would simply fix one or another of the metastable magnetic configurations at no additional energy cost. One can see that, indeed, a common band gap can be found in all situations relevant for our discussion, i.e. with FSM $=1,5$, and $15 \mu_{\mathrm{B}}$, thus justifying the extraction of the $J$ value above. Another observation concerns the local magnetic moments at Fe sites: as the FSM value is changing, and even as the saturation of magnetization (at FSM $=15 \mu_{\mathrm{B}}$ ) is achieved, the local Fe magnetic moments merely flip, essentially maintaining their magnitudes of $4 \mu_{\mathrm{B}}$, and not $5 \mu_{\mathrm{B}}$. The nominal value of $s=5 / 2$, associated with a single Fe atom, comes about due to magnetic polarization of ligands. This situation is identical to that described earlier for "ferric wheels" $[3,8,10]$. In spite of the very different structure of organic ligands, both systems have similarities in the nearest neighbourhood and in the charge state of $\mathrm{Fe}$ atoms $\left(\mathrm{O}_{6}\right.$ twisted octahedral coordination in "ferric wheels" vs. slightly distorted octahedral $\mathrm{O}_{5} \mathrm{~N}$ coordination in the present system).

\section{Fe-binuclear system: Effect of intraatomic correlation}

We turn now to the discussion of the effect of on-site intraatomic Coulomb correlation on interatomic exchange parameters. Our objective was to find a compact and yet realistic system, for which an electronic structure calculation could be done with the use of an ultimately accurate method within the DFT, that is, the FLAPW method, and to apply a phenomenological correction by the $\mathrm{LDA}+U$ formalism [11] on top of it. Specifically, we apply the realization of the method in the FLEUR code [6]. We derive a simple model system from the Fe-binuclear complex described by Real et al. [12] being subject to previous studies [13]. We neglect for the moment the spin crossover property, which makes this system particularly interesting, and make our calculation for the high-spin configuration on both Fe centers. The chemical formula of the substance is $\left[\mathrm{Fe}(\mathrm{bt})(\mathrm{NCS})_{2}\right]_{2}$-bpym, with bt $=2,2^{\prime}$-bithazoline and bpym $=2,2^{\prime}$-bipyrimidine, and the molecular unit as shown on the left of Fig. 2. We also performed electronic structure calculation for this substance in its molecular and crystallized form by the SIESTA method, allowing structure relaxation, the results of this study will be reported elsewhere. For the present simulation, as the molecule is yet too large and too low-symmetric for an affordable FLAPW calculation, we simplify the molecular units, substituting the bt fragments by bpym - that is realistic, as this is encountered in related compounds - see, e.g., [13]. Moreover we cut and connect the (NCS) tails into $-\mathrm{N}=\mathrm{C}=\mathrm{N}-$ chains that makes a dense 3-dimensional lattice out of planar $\mathrm{Fe}-$ bipyrimidine groups (Fig. 2, right panel). The nearest neighbourhood of each Fe center remains a slightly distorted $\mathrm{N}_{6}$ octahedron, as was the case in the original molecular unit. An idealization is that all $\mathrm{N}-\mathrm{Fe}-\mathrm{N}$ bonds make now right angles, and the repeated bipyrimidine fragments are coplanar. 

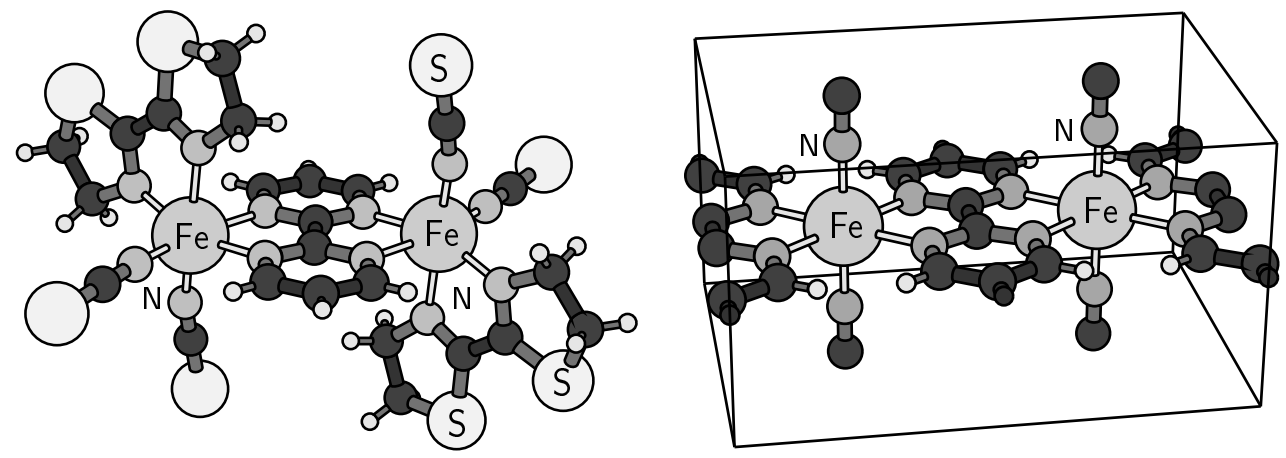

Fig. 2. Molecular unit of the Fe-binuclear system Fe[(bt)(NCS $\left.)_{2}\right]_{2}$ bpym described in Ref. [12] (left) and a simplified model thereof, used in the calculation (right). The Fe atom is in the middle of a slightly distorted $\mathrm{N}_{6}$ octahedron.

The calculated densities of states (per unit cell and per one Fe site) in Fig. 3 clearly indicate a hybridization of Fe3d with the $2 p$ states of neighbouring nitrogen atoms. Since the minority-spin Fe $3 d$ states are not completely empty, the local magnetic moment is less than $5 \mu_{\mathrm{B}}$. As for the previous trinuclear system, the magnetic moment induced on nitrogen neighbours substantially contributes to the net value, associated to each Fe site. This resolves, at least in part, a controversy between the spin value $s=5 / 2$, expected at the Fe site from formal valence considerations, and the local magnetic moment of our calculation. The cumulative magnetic moment, distributed over the Fe site and its neighbours, behaves like a "rigid" spin in the sense of the Heisenberg model. However, one can expect that a stronger localization of this distributed magnetic moment at the Fe site will be enforced by an inclusion of intraatomic Coulomb correlation, underestimated in conventional DFT calculations. In order to check this possible effect, we brought in an additional Coulomb correlation applying a semi-empirical
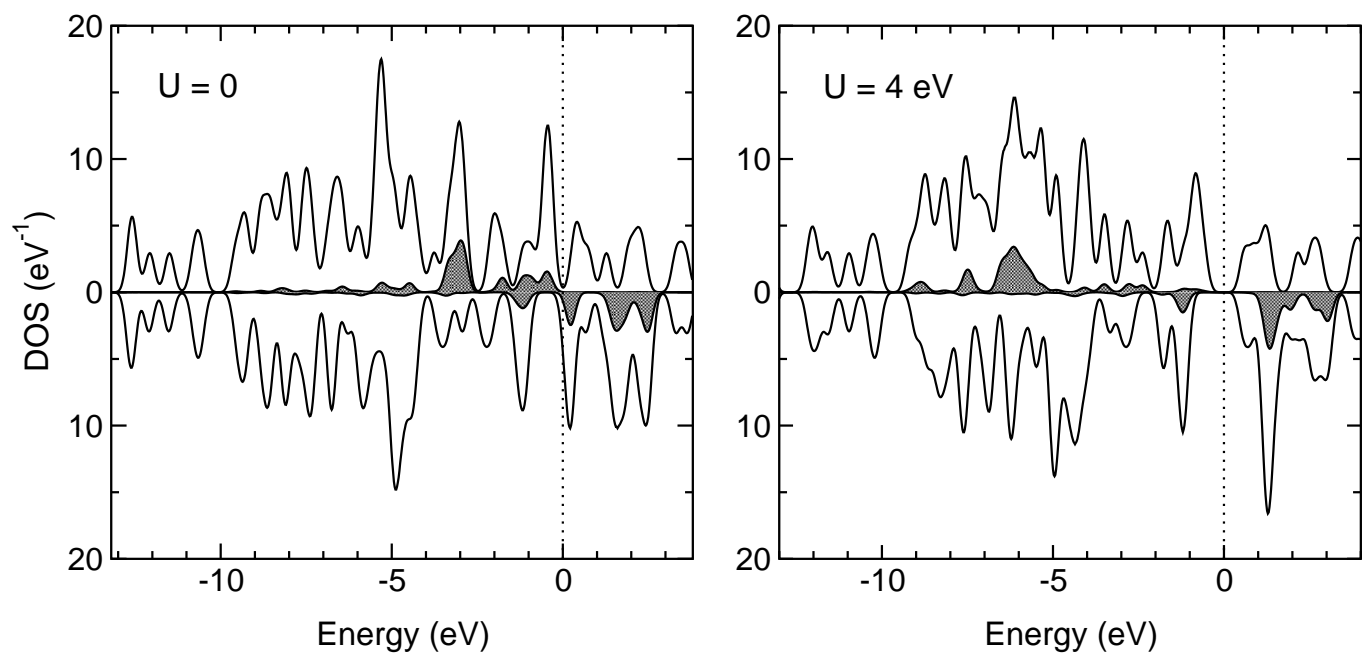

Fig. 3. Total density of states per unit cell (outer solid line) and local DOS per Fe site (evaluated within the muffin-tin sphere of 2.3 Bohr; shown by filled black) densities of states calculated for the model Fe-binuclear system, shown in the right panel of Fig. 2, for parallel orientation of Fe magnetic moments. Left panel: LDA calculation, right panel: $\mathrm{LDA}+U$ calculation with $U=4 \mathrm{eV}$. 
Table 1

Local magnetic moments at the Fe site (in $\mu_{\mathrm{B}}$, within muffin-tin sphere of 2.3 Bohr); total magnetic moments per Fe center, total energy difference between FM and AFM configurations, and corresponding extracted values of the Heisenberg exchange parameter $J$, assuming nominal spin value $s=5 / 2$.

\begin{tabular}{|c|c|c|c|c|c|}
\hline \multirow[b]{2}{*}{$U(\mathrm{eV})$} & \multicolumn{2}{|c|}{$M_{\mathrm{Fe}}$} & \multirow{2}{*}{$\begin{array}{c}M / \mathrm{Fe} \\
\mathrm{FM}\end{array}$} & \multirow[b]{2}{*}{$E_{\mathrm{FM}}-E_{\mathrm{AFM}}(\mathrm{meV})$} & \multirow[b]{2}{*}{$J(\mathrm{~K}$} \\
\hline & $\mathrm{FM}$ & $\mathrm{AFM}$ & & & \\
\hline 0 & 3.62 & 3.61 & 4.10 & 102.5 & -95 \\
\hline 4 & 3.93 & 3.92 & 4.94 & 76.8 & -71 \\
\hline
\end{tabular}

"LDA $+U$ " scheme [11]. We choose the Coulomb interaction constant $U=4 \mathrm{eV}$, which seems reasonable for correlated Fe compounds [14,15]. One sees in the DOS of Fig. 3 (right panel) the known effect of the " $+U$ " correction to lower the occupied states in energy whereas shifting unoccupied ones upwards.

The values of magnetic moments and exchange parameters $J$, determined by the formula $E=-J \mathbf{s}_{1} \mathbf{s}_{2}$, are listed in the Table. It is noteworthy that, whereas the total magnetic moment per Fe site is increased in the LDA $+U$ calculation, the local Fe magnetic moment still does not exceed $4 \mu_{\mathrm{B}}$. On can conclude that the spatial distribution of magnetic density is not confined to the Fe atom even as the intraatomic Coulomb correlation is enhanced; instead, the magnetization maintains its complex spatial distribution, spilling onto neighbouring $\mathrm{N}$ atoms.

As the intraatomic Coulomb correlation shifts apart on the energy scale the centers of gravity of occupied and unoccupied states, it reduces the interatomic exchange parameter, because the latter (in one of its formulations, see [16]) has corresponding energy differences in the denominator. The same trend prevails in our case even as we estimate the values of $J$ by comparing the total energies of two magnetic configurations. The effect of Coulomb correlation, included on top of a straightforward DFT calculation, is therefore always towards lowering the absolute values of Heisenberg exchange parameters. The same conclusion has been drawn by Boukhvalov et al. in their calculations for " $\mathrm{Mn}_{12}$ " [17] and " $\mathrm{V}_{15}$ " [18] molecular magnets. Comparing with the results of the previous Section, one can conclude that the estimation of exchange parameters in molecular magnets, that is already qualitatively correct (in sign and order of magnitude) in a conventional DFT calculation, can be brought closer to the experimental estimates by reducing their numerical values due to additionally included intraatomic Coulomb correlation.

\section{Acknowledgements}

The authors thank Deutsche Forschungsgemeinschaft for financial support (Priority Program 'Molecular Magnetism'). A.V.P. thanks Colette Boskovic and José 
Real for providing crystallographic information on substances synthesized by them, and Danil Boukhvalov for making accessible his manuscript on $V_{15}$ prior to publication.

\section{References}

[1] O. Kahn, Molecular Magnetism, John Wiley \& Sons, Singapore, 1993.

[2] J. Kortus, A. V. Postnikov, Molecular Nanomagnets, to be published in: M. Rieth, W. Schommers (Eds.), Handbook of Theoretical and Computational Nanotechnology, Vol. 10 of Encyclopedia of Nanoscience and Nanotechnology, American Scientific Publishers (2004) http://aspbs.com/html/a0000tcn.htm

[3] A. V. Postnikov, J. Kortus, M. R. Pederson, Density functional studies of molecular magnets, Scientific Highlight of the month February 2004, Newsletter 61 of the $\Psi_{k^{-}}$ Network, http://psi-k.dl.ac.uk/newsletters/News_61/Highlight_61.pdf.

[4] SiESTA homepage, http://www.uam.es/siesta.

[5] J. M. Soler, E. Artacho, J. D. Gale, A. García, J. Junquera, P. Ordejón, D. SánchezPortal, J. Phys.: Condens. Matter 14 (2002) 2745.

[6] FLEUR homepage, http://www.flapw.de.

[7] C. Boskovic, A. Sieber, G. Chaboussant, H. U. Güdel, J. Ensling, W. Wernsdorfer, A. Neels, G. Labat, H. Stoeckli-Evans, S. Janssen, Inorg. Chem. 43 (2004) 5053.

[8] A. V. Postnikov, S. G. Chiuzbăian, M. Neumann, S. Blügel, J. Phys. Chem. Solids $65(2004) 813$.

[9] K. Schwarz, P. Mohn, J. Phys. F: Metal Phys. 14 (1984) L129.

[10] A. V. Postnikov, J. Kortus, S. Blügel, Molec. Phys. Rep. 38 (2003) 56; http://arXiv.org/abs/cond-mat/0307292.

[11] V. I. Anisimov, F. Aryasetiawan, A. I. Lichtenstein, J. Phys.: Condens. Matter 9 (1997) 767.

[12] J.-A. Real, H. Bolvin, A. Bousseksou, A. Dworkin, O. Kahn, F. Varret, J. Zarembowitch, J. Am. Chem. Soc. 114 (1992) 4650.

[13] J.-F. Létard, J. A. Real, N. Moliner, A. B. Gaspar, L. Capes, O. Cador, O. Kahn, J. Am. Chem. Soc. 121 (1999) 10630.

[14] I. I. Mazin, V. I. Anisimov, Phys. Rev. B 55 (1997) 12822.

[15] M. P. J. Punkkinen, K. Kokko, W. Hergert, I. J. Väyrynen, J. Phys.: Condens. Matter 11 (1999) 2341.

[16] A. I. Liechtenstein, V. I. Anisimov, J. Zaanen, Phys. Rev. B 52 (1995) R5467.

[17] D. W. Boukhvalov, A. I. Lichtenstein, V. V. Dobrovitski, M. I. Katsnelson, B. N. Harmon, V. V. Mazurenko, V. I. Anisimov, Phys. Rev. B 65 (2002) 184435. 
[18] D. W. Boukhvalov, V. V. Dobrovitski, M. I. Katsnelson, A. I. Lichtenstein, B. N. Harmon, P. Kögerler, to be published in Phys. Rev. B (2004).

[19] It should be explained that the Fe-trinuclear complex reported in Ref. [7] crystallizes with two molecular units per primitive cell, which are slightly different. In particular, there is a formal disproportionality in the nominal electron number between these two fragments. As a consequence, a single molecular unit we took for our calculation possesses an odd number of electrons. Therefore only odd FSM values correspond to integer number of electrons in both majority- and minorityspin channels and may lead to an appearance of HOMO-LUMO gaps, shown in the middle panel of Fig. 1. However, the even FSM values can be formally applied in the calculation as well, notwithstanding that they do not possess the HOMOLUMO gap. Technically we applied an artificial broadening of molecular orbitals' eigenvalues by $400 \mathrm{~K}$. Some of the even FSM numbers allow an easily converging calculation, with the total energy and local moments which follow the general trend. Such results are added in the left and right panels of Fig. 1. 